\title{
Estrategias didácticas en la enseñanza de los productos notables y la factorización en la telesecundaria
}

\author{
Teaching Strategies in the Teaching of Notable Products and Factoring in \\ the Telesecundaria
}

\begin{abstract}
Estratégias didáticas no ensino de produtos notáveis e fatoração em telesecundária

Enrique Gómez Segura

Escuela Normal Urbana Federal Profr. Rafael Ramírez, México

egos72@hotmail.com

https://orcid.org/0000-0002-3730-4556
\end{abstract}

\section{Resumen}

El presente trabajo tiene el objetivo de resignificar las operaciones matemáticas de los productos notables y la factorización con procedimientos geométricos y razonamiento lógico matemático, de tal manera que los estudiantes vean las operaciones como inversas una de otra y esto les permita transitar matemáticamente con mayor facilidad. Este contenido matemático se estudia en el tercer grado de educación telesecundaria, en el primer bloque del programa. Para aplicar las actividades didácticas, se rescataron los conocimientos previos de los estudiantes, lo cual les permitió construir con mayor facilidad las reglas para el desarrollo de los productos notables como es el caso de los binomios cuadrados, conjugados y binomios con un término común; posteriormente, se realizaron las actividades inversas, es decir, pasar de un trinomio cuadrado perfecto a un binomio cuadrado y así sucesivamente con los otros dos productos notables. En cuanto a los resultados obtenidos por la implementación de las secuencias didácticas, 17 alumnos $(85 \%)$ tuvieron un mejor aprovechamiento escolar. En conclusión, los alumnos pudieron relacionar las operaciones en forma retrospectiva hasta 


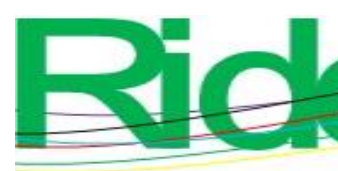

Revista Iberoamericana para la
Investigación y el Desarrollo Educativo
ISSN $2007-7467$

llegar al punto de partida; este razonamiento casi desperdiciado en la enseñanza de la matemática permite apropiarse de la resolución de problemas con su respectiva comprobación, eso quiere decir que existe un aprendizaje con un razonamiento metacognitivo. Las únicas dificultades que se observaron en el aprendizaje se presentaron en la representación gráfica de los binomios conjugados y en la construcción de la regla de los binomios con un término común.

Palabras clave: plan de estudios, resolución de problemas, secuencia didáctica.

\section{Abstract}

The present work has the objective of resignifying the mathematical operations of the notable products and the factorization with geometric procedures and mathematical logical reasoning, in such a way that the students see the operations as inverses of each other and this allows them to move mathematically with greater ease. This mathematical content is studied in the third grade of telesecundaria education, in the first block of the program. To apply the didactic activities, the previous knowledge of the students was rescued, which allowed them to more easily build the rules for the development of notable products, such as the case of square binomials, conjugates and binomials with a common term; subsequently, the inverse activities were carried out, that is, going from a perfect square trinomial to a square binomial and so on with the other two notable products. Regarding the results obtained by the implementation of the didactic sequences, 17 students $(85 \%)$ had a better school performance. In conclusion, the students were able to relate the operations retrospectively until they reached the starting point; this almost wasted reasoning in the teaching of mathematics allows appropriating the resolution of problems with their respective verification, that means that there is learning with metacognitive reasoning. The only difficulties observed in learning were presented in the graphical representation of the conjugate binomials and in the construction of the binomial rule with a common term.

Keywords: curriculum, problem solving, didactic sequence. 


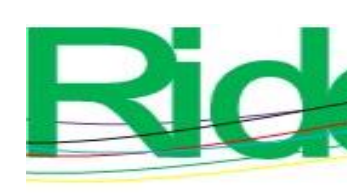

Revista Iberoamericana para la
Investigación y el Desarrollo Educativo
ISSN $2007-7467$

\section{Resumo}

O presente trabalho tem o objetivo de ressignificar as operações matemáticas dos produtos notáveis e a fatoração com procedimentos geométricos e raciocínio lógico matemático, de tal forma que os alunos vejam as operações como inversas umas das outras e isso lhes permita se mover matematicamente com maior facilidade. Esse conteúdo matemático é estudado na terceira série do ensino telesecundário, no primeiro bloco do programa. Para aplicar as atividades didáticas, foi resgatado o conhecimento prévio dos alunos, o que lhes permitiu construir com mais facilidade as regras para o desenvolvimento de produtos notáveis, como é o caso de binômios quadrados, conjugados e binômios com termo comum; Posteriormente, foram realizadas as atividades inversas, ou seja, passar de um trinômio quadrado perfeito para um binômio quadrado e assim sucessivamente com os outros dois produtos notáveis. Em relação aos resultados obtidos com a aplicação das sequências didáticas, 17 alunos (85\%) obtiveram melhor aproveitamento acadêmico. Em conclusão, os alunos conseguiram relacionar as operações retrospectivamente até chegarem ao ponto de partida; Esse raciocínio quase desperdiçado no ensino de matemática permite apropriar-se da resolução de problemas com sua respectiva verificação, ou seja, há aprendizagem com raciocínio metacognitivo. As únicas dificuldades observadas na aprendizagem foram apresentadas na representação gráfica dos binômios conjugados e na construção da regra binomial com um termo comum.

Palavras-chave: currículo, resolução de problemas, sequência didática.

Fecha Recepción: Julio 2021

Fecha Aceptación: Enero 2022

\section{Introducción}

Es importante que los estudiantes puedan transitar por diversos registros matemáticos. Tal y como lo señala Duval (2016), desde el punto de vista cognitivo, hay diferentes representaciones semióticas y nunca debe confundirse el objeto matemático con su representación semiótica. Por ello, el objetivo del presente trabajo de investigación es resignificar las operaciones matemáticas de los productos notables y la factorización con procedimientos geométricos y razonamiento lógico matemático.

Gómez (2015) parte del hecho de que "la factorización es un contenido del curso de matemática, de segundo básico, que presenta dificultades en su aprendizaje, lo que afecta el desarrollo de los estudiantes en la asimilación de temas posteriores" (p. 25). Esto mismo 


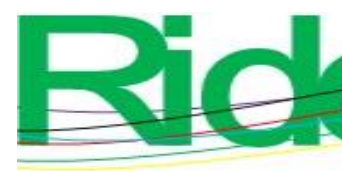

Revista Iberoamericana para la Investigación y el Desarrollo Educativo ISSN 2007 - 7467

sucede en la Escuela Normal Urbana Federal Profr. Rafael Ramírez de la ciudad de Chilpancingo, Guerrero, México, la cual oferta la licenciatura en Educación Secundaria con especialidad en Telesecundaria.

En México, el programa de estudios diseñado por la Secretaría de Educación Pública [SEP] (2017) establece que el tópico "Productos notables y factorización" (p. 313) sea impartido en tercer grado de educación secundaria. Dicho tema no es de fácil tratamiento didáctico. Normalmente, los alumnos tienen dificultades para asimilarlo. Parte de su complejidad se debe a la estructura organizativa de los programas, falta de actualización de los profesores que imparten dicha asignatura y carencia de trabajo cooperativo entre los docentes. Según Méndez y Cruz (2008):

Uno de los conceptos que en el proceso de enseñanza-aprendizaje no llegan a dominar los alumnos, durante gran parte de la enseñanza media, es el desarrollo de identidades notables y la factorización. Muchos de ellos no tienen éxito al resolver ejercicios, ni ponen en obra los procedimientos correctos cuando se enfrentan a situaciones en las que este concepto es parte de la solución. El estudio de esta problemática evidencia un fenómeno didáctico complejo (p. 59).

En cuanto a la corriente constructivista, Rico (1998) menciona que:

- Todo conocimiento es construido. El conocimiento matemático es construido, al menos en parte, a través de un proceso de abstracción reflexiva. - Existen estructuras cognitivas que se activan en los procesos de construcción.

- Las estructuras cognitivas están en desarrollo continuo. La actividad con propósito induce la transformación de las estructuras existentes.

- Reconocer el constructivismo como una posición cognitiva conduce a adoptar el constructivismo metodológico (pp. 74-75).

El diseño didáctico siempre busca que los alumnos observen, analicen, construyan, comuniquen, argumenten y resuelvan los problemas planteados en los temas ya mencionados. Es importante mencionar que el profesorado puede consultar textos de educación preescolar, primaria, secundaria y nivel medio superior en la página de la Comisión Nacional de Libros de Textos Gratuitos de México 


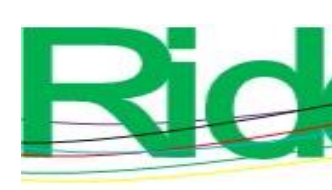

Revista Iberoamericana para la
Investigación y el Desarrollo Educativo
ISSN $2007-7467$

(https://www.conaliteg.sep.gob.mx/secundaria.html). Especialmente, para la enseñanza de las matemáticas a nivel secundaria se recomienda consultar a Ángeles, Guerrero y Loyola (2013) .

El tema aquí tratado corresponde a una rama en particular de las matemáticas.

El álgebra tiene una gran presencia como contenido matemático en diferentes etapas en el sistema educativo, especialmente desde la secundaria obligatoria hasta la universidad, aunque en los últimos 20 años han surgido propuestas de incorporar ciertas cuestiones del pensamiento algebraico en la educación primaria (Socas, 2011, p. 5).

Generalmente, estos temas se trabajan de manera tradicional en la escuela secundaria y en el bachillerato. En palabras de Navarro (2018), "de profesores que transmiten y de alumnos que reciben información" (p. 195). El docente ofrece las fórmulas de la factorización para que los alumnos sustituyan los valores en estas y obtengan los resultados correspondientes. Esta acción, sin embargo, no les permite construir las reglas de cada binomio, por lo tanto, cada vez que requieren hacer uso de estas, tienen que ver un formulario de productos notables y factorización.

Aquí, por el contrario, se busca que los alumnos sean activos, no pasivos, que ellos sean los que promuevan ideas de resolución y que no tengan que esperar la explicación del profesor sobre el procedimiento que deberán seguir para resolver el problema (del Carmen, Alfonzo y Trejo, 2016). En esa línea, el docente debe cumplir más bien el rol de facilitador del aprendizaje, no de trasmisor del conocimiento.

La factorización se inicia en la educación primaria (Alarcón, 2005): cuando se calcula el mínimo común múltiplo en las operaciones de suma y resta de fracciones heterogéneas y también cuando se calcula el máximo común divisor. En la escuela secundaria, este contenido se tiene reservado para el tercer grado (Ángeles, 2013)

La pregunta de investigación de este trabajo es la siguiente: ¿construirán los estudiantes las reglas de los productos notables con las actividades didácticas diseñadas? Y la hipótesis es que los estudiantes pueden construir las reglas de los productos notables si analizan cada una de sus partes como multiplicaciones de las operaciones algebraicas. 


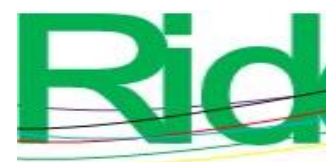

Revista Iberoamericana para la
Investigación y el Desarrollo Educativo
ISSN $2007-7467$

Metodología

La investigación es cualitativa, exploratoria y explicativa. Se trata de conocer el comportamiento de los educandos, clarificar los problemas de enseñanza-aprendizaje de los productos notables y la factorización y explorar y explicar las causas de la carencia del dominio integral de los contenidos para que, posteriormente, se tomen las medidas pertinentes.

Para la elaboración de la secuencia didáctica, se siguió el siguiente procedimiento:

a) Se revisaron los textos de telesecundaria, secundaria general, técnicas y de nivel medio superior (telebachillerato).

b) A través de Google y Google Académico, se consultaron libros en formato digital que pudieran dar mayor información sobre el tema en cuestión.

c) Los apuntes de clase y planeaciones para tratar dicho contenido.

d) Se diseñó un posible índice para dar seguimiento a las actividades.

e) Fueron seleccionados algunos softwares graficadores para apoyar al diseño de la secuencia didáctica.

f) Se diseñó y aplicó la secuencia didáctica.

g) Se valoraron los resultados de la aplicación.

h) Se informa en el presente documento.

\section{Productos notables \\ Conocimientos previos}

- Actividad 1. Escribe los elementos de las siguientes operaciones 


\section{Revista Iberoamericana para la Investigación y el Desarrollo Educativo ISSN 2007 - 7467}

Figura 1. Elementos de las operaciones aritméticas
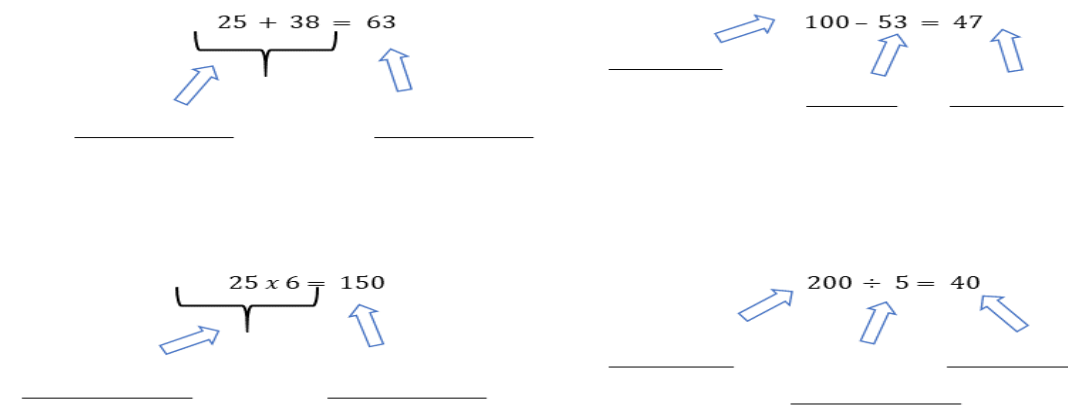

Fuente: Elaboración propia

\section{Leyes de los exponentes}

Actividad 2. Con la fórmula de multiplicación $x^{m} x^{n}=x^{m+n}$, realiza las siguientes expresiones algebraicas:

$$
x^{12} \cdot x^{3}, x^{9} \cdot x^{-15}, x^{7} \cdot x^{8}, x^{2} \cdot x^{-3}, x^{-5} \cdot x^{-4}, x^{2} \cdot x^{3}, x^{-6} \cdot x^{-9}, x^{8} \cdot x^{-3}, x^{8} \cdot x^{-3} y x^{20} \cdot x^{-9}
$$

Actividad 3. Con la fórmula $\frac{x^{m}}{x^{n}}=x^{m-n}$, realiza las siguientes divisiones:

$$
\frac{x^{12}}{x^{5}}, \frac{x^{10}}{x^{6}}, \frac{x^{-2}}{x^{-4}}, \frac{x^{13}}{x^{-8}}, \frac{x^{16}}{x^{-6}}, \frac{x^{-12}}{x^{-20}}, \frac{x^{28}}{x^{50}}, \frac{x^{19}}{x^{5}}, \frac{x^{23}}{x^{7}} y \frac{x^{-7}}{x^{4}}
$$

Actividad 4. A través de la fórmula $x^{-n}=\frac{1}{x^{n}}$, cambia las potencias negativas a positivas de las siguientes expresiones:

$$
x^{-2}, x^{-5}, x^{-9}, x^{-1}, x^{-6}, x^{-15}, x^{-12}, x^{-8}, x^{-20} \text { y } x^{-17}
$$

Actividad 5. Con la fórmula $\left(x^{n}\right)^{m}=x^{m n}$, realiza las siguientes operaciones de potencia:

$$
\left(x^{2}\right)^{3},\left(x^{3}\right)^{5},\left(x^{-2}\right)^{-6},\left(x^{-7}\right)^{4},\left(x^{5}\right)^{3},\left(x^{12}\right)^{-5},\left(x^{-6}\right)^{-4},\left(x^{\frac{2}{5}}\right)^{6},\left(x^{\frac{3}{4}}\right)^{-7} y\left(x^{\frac{5}{6}}\right)^{2}
$$




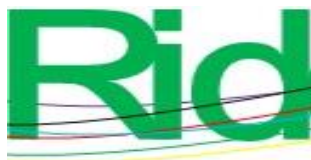

Revista Iberoamericana para la Investigación y el Desarrollo Educativo ISSN 2007 - 7467

\section{Binomio cuadrado}

Actividad 6. Encuentra el área de los siguientes cuadrados por partes coloreadas

Figura 2. Cuadrados de diferentes tamaños
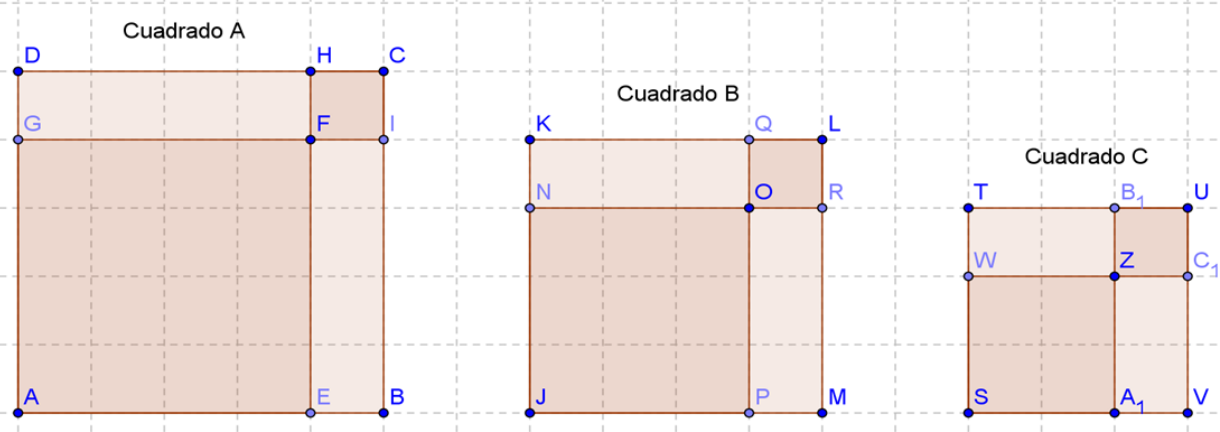

Fuente: Elaboración propia

Área del cuadrado A: Área del cuadrado B: Área del cuadrado C:

Actividad 7. Calcula el área que tiene sus lados en términos algebraicos.

Figura 3. Cuadrados con medidas algebraicas
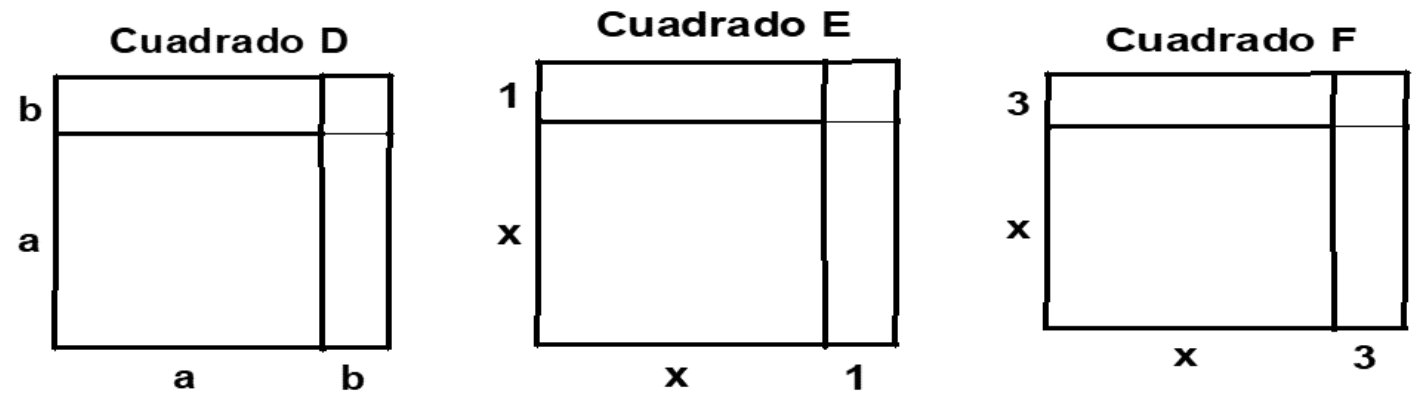

Fuente: Elaboración propia

Área: Área: Área: 


\section{Revista Iberoamericana para la Investigación y el Desarrollo Educativo ISSN 2007 - 7467}

Actividad 8. Revisando patrones de regularidad, tenemos los siguientes productos, paso por paso:

- Cuadrado D. $(a+b)(a+b)=a^{2}+a b+a b+b^{2}=a^{2}+2 a b+b^{2}$

- Cuadrado E. $(x+1)(x+1)=x^{2}+x+x+1^{2}=x^{2}+2 x+1$

Para analizar, vamos a simplificar los binomios de la siguiente manera:

$(a+b)(a+b)=(a+b)^{2},(x+1)(x+1)=(x+1)^{2}$ y $(x+3)(x+3)=(x+3)^{2}$

Ahora tenemos lo siguiente con respecto al cuadrado D: el producto de los lados se representa como $(a+b)^{2}$, cuyo producto es $a^{2}+2 a b+b^{2}$

¿Cómo se obtienen los siguientes términos algebraicos?

- $\quad$ El primer término:

- $\quad$ El segundo término:

- $\quad$ El tercer término:

Ahora tenemos lo siguiente con respecto al cuadrado E: el producto de los lados se representa como $(x+1)^{2}$, cuyo producto es $x^{2}+2 x+1$

¿Cómo se obtienen los siguientes términos algebraicos?

- $\quad$ El primer término:

- $\quad$ El segundo término:

- $\quad$ El tercer término:

Ahora tenemos lo siguiente con respecto al cuadrado F: el producto de los lados se representa como $(x+3)^{2}$, cuyo producto es $x^{2}+6 x+9$

¿Cómo se obtienen los siguientes términos algebraicos?

- $\quad$ El primer término:

- $\quad$ El segundo término:

- $\quad$ El tercer término:

Escribe una regla para obtener el producto de un binomio al cuadrado: "Es el cuadrado del ". 

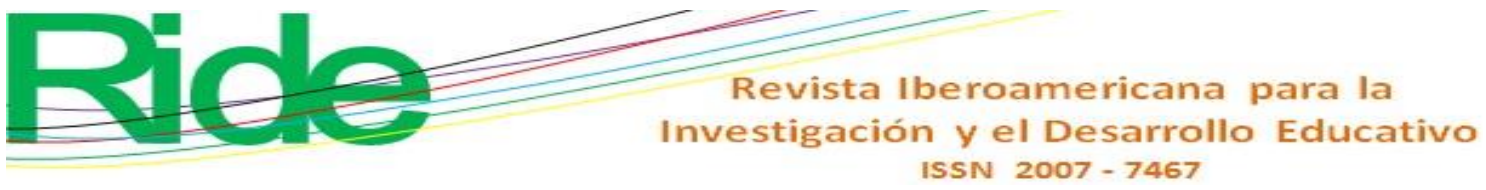

Actividad 9. Con la regla obtenida, resuelve los siguientes binomios cuadrados:

$$
(x+5)^{2},(x-2)^{2},(x+9)^{2},(2 x+4)^{2},(3 x-2)^{2},(3 x+7)^{2},(5 x+10)^{2},(5 x+10)^{2}
$$

\section{Binomios conjugados}

Actividad 10. Contesta las siguientes preguntas:

- ¿Cuáles son los números simétricos?

- ¿Cuáles son los binomios conjugados?

Actividad 11. Determina el área sombreada del color más fuerte de las siguientes figuras geométricas.

Figura 4. Cuadrados para binomios conjugados

Figura $\mathrm{G}$

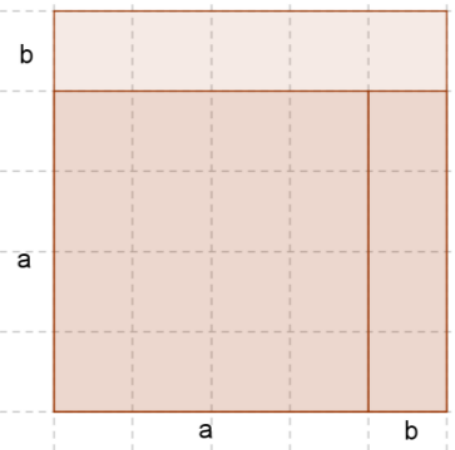

Figura $\mathrm{H}$

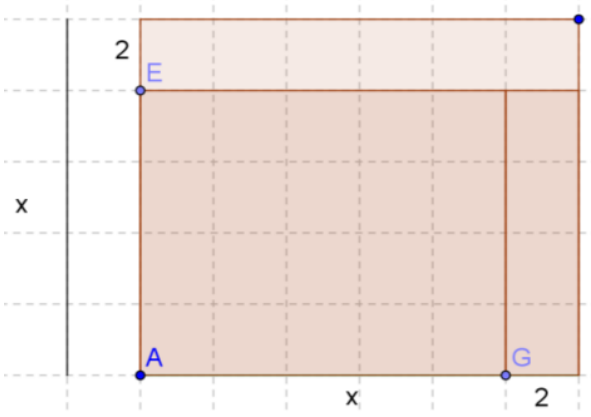

Fuente: Elaboración propia

- ¿QQué expresión algebraica le corresponde a la base de la figura G?

- ¿Qué expresión algebraica le corresponde a la altura de la figura G?

- ¿Cuál es la expresión algebraica en términos de área de la figura G?

- ¿Qué expresión algebraica le corresponde a la base de la figura $\mathrm{H}$ ?

- ¿Qué expresión algebraica le corresponde a la altura de la figura H?

- ¿Cuál es la expresión algebraica en términos de área de la figura H? 


\section{Revista Iberoamericana para la Investigación y el Desarrollo Educativo ISSN 2007 - 7467}

Actividad 12. Revisando patrones de regularidad, tenemos los siguientes productos, paso por paso:

- Cuadrado D. $(a+b)(a-b)=a^{2}-a b+a b-b^{2}=a^{2}-b^{2}$

- $\quad$ Cuadrado E. $(x+2)(x-2)=x^{2}-2 x+2 x-4=x^{2}-4$

De la figura $G$ tenemos las siguientes observaciones: sus lados miden $(a+b)(a-b)$, tienen un término común (a), tiene dos términos simétricos $(b y-b)$, su producto se compone de una diferencia de dos términos cuadráticos, uno de ellos es el término común $\left(a^{2}\right)$ y el otro es el término simétrico $\left(b^{2}\right)$.

¿Cómo se obtienen los dos primeros términos algebraicos?

De la figura $\mathrm{H}$ tenemos las siguientes observaciones: sus lados miden $(x+2)(x-2)$, tienen un término común $(x)$, tiene dos términos simétricos $(2 y-2)$, su producto se compone de una diferencia de dos términos cuadráticos, uno de ellos es el término común $\left(x^{2}\right)$ y el otro es el término simétrico (4).

¿Cómo se obtienen los dos primeros términos algebraicos?

Escribe una regla para obtener el producto de los binomios conjugados: "Es el cuadrado del

Actividad 13. Grafica en tu cuaderno los siguientes binomios conjugados y encuentra el área de cada uno de ellos, aplicando solamente la regla obtenida. Estos son:

$$
(x+1)(x-1),(x+3)(x-3),(x+5)(x-5),(x+8)(x-8),(x+10)(x-10)
$$




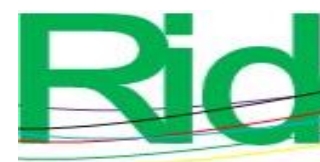

Revista Iberoamericana para la Investigación y el Desarrollo Educativo ISSN 2007 - 7467

\section{Binomios con un término común}

- ¿QQué se entiende por binomio y por común en términos matemáticos?

Actividad 14. Determina los lados y el área de cada una de las partes que conforman las siguientes figuras geométricas.

Figura 5. Cuadrados que conforman binomios con un término común
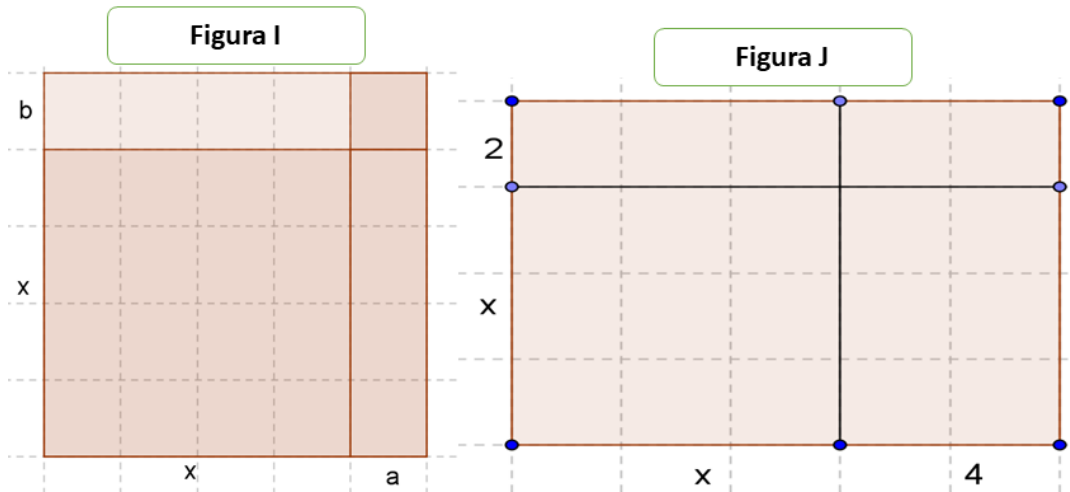

Fuente: Elaboración propia con base en Steven (2013)

- ¿ ¿Qué expresión algebraica le corresponde a la base de la figura I?

- ¿ ¿Qué expresión algebraica le corresponde a la altura de la figura I?

- ¿ ¿Cuál es la expresión algebraica en términos de área de la figura I?

- ¿ ¿Qué expresión algebraica le corresponde a la base de la figura J?

- ¿ ¿Qué expresión algebraica le corresponde a la altura de la figura J?

- ¿ ¿Cuál es la expresión algebraica en términos de área de la figura J?

De acuerdo con lo observado, podemos concluir que el producto de dos binomios con un término común se obtiene de la siguiente manera:

- $\quad$ El primer término:

- $\quad$ El segundo término:

- $\quad$ El tercer término:

Escribe una regla para obtener el producto de los binomios con un término común: "Es el cuadrado del , 


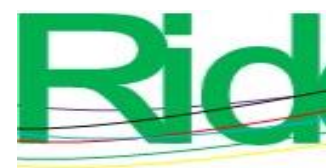

Revista Iberoamericana para la
Investigación y el Desarrollo Educativo
ISSN $2007-7467$

Actividad 15. Grafica en tu cuaderno los siguientes binomios con un término común y encuentra el área de cada uno de ellos, aplicando solamente la regla obtenida. Los binomios son los siguientes:

$(x+2)(x+1),(x+3)(x+5),(x+5)(x+4),(2 x+8)(2 x+1),(3 x+10)(3 x-4)$

\section{La factorización de las expresiones algebraicas El trinomio cuadrado perfecto}

Actividad 16. Contesta las siguientes interrogantes:

- ¿Qué se entiende por factorización?

- ¿ ¿De qué binomios se generan los trinomios cuadrados perfectos?

Ahora sabemos que a un trinomio cuadrado perfecto factorizado le corresponde un binomio cuadrado. Un ejemplo:

a) $\quad a^{2}+2 a b+b^{2}=(a+b)^{2}$

- ¿Cómo se obtiene el primer término del binomio cuadrado?

- ¿Cómo se obtiene el segundo término del binomio cuadrado?

- ¿ ¿Cómo se obtiene el signo que separa a las raíces obtenidas?

Resumen del procedimiento: dado el trinomio cuadrado perfecto, se procede de la siguiente manera:

$$
a^{2}+2 a b+b^{2}
$$

Paso 1. Se extrae raíz cuadrada a los términos cuadráticos.

$$
\sqrt{a^{2}}=a, \sqrt{b^{2}}=b
$$

Paso 2. Ambas raíces son separadas por el signo del segundo término del trinomio.

Paso 3. Se encierra en un paréntesis las dos raíces separadas con el signo del segundo término del trinomio cuadrado perfecto y se eleva al cuadrado.

Paso 4. La expresión queda de la siguiente manera:

$$
(a+b)^{2}
$$


Actividad 17. Factoriza los siguientes trinomios (identifica cuáles son):

$$
4 x^{2}+24 x+36,25 x^{2}-20 x+4,9 x^{2}+24 x+16,49 x^{2}+29 x+4
$$

\section{La diferencia de cuadrados}

Actividad 18. Contesta las siguientes interrogantes:

- ¿Qué entiendes por diferencia?

- ¿Qué entiendes por cuadrados?

Fíjate en las siguientes igualdades:

$$
(a+b)(a-b)=a^{2}-b^{2}
$$

- ¿Cómo crees que se obtiene el término común $(a)$ ?

- ¿Cómo crees que se obtiene el término simétrico $(b$ y $-b)$ ?

De acuerdo con lo que mencionaste, escribe una regla para pasar de una diferencia de cuadrados a dos binomios conjugados: "La raíz cuadrada del ".

Actividad 19. Utilizando la regla antes descrita, factoriza las diferencias de cuadrados de las siguientes expresiones algebraicas:

$$
x^{2}-z^{2}, 9 x^{2}-16 y^{2}, 49 x^{2}-64,4 x^{2}-81,25 x^{2}-36
$$




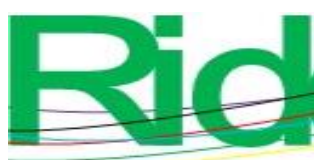

Revista Iberoamericana para la Investigación y el Desarrollo Educativo ISSN 2007 - 7467

\section{Binomios con un término común}

Actividad 20. Contesta las siguientes interrogantes:

- ¿Recuerda cómo se obtiene un trinomio de segundo grado?

$$
(x+3)(x+4)=x^{2}+7 x+12
$$

El primer término se le llama término cuadrático $\left(x^{2}\right)$, el segundo se llama término lineal $(7 \mathrm{x})$ y el tercero término independiente o constante (12); mientras que los elementos de los binomios son: término común (x) y términos no comunes (3 y 4)

Contesta las siguientes interrogantes

- Con los elementos del trinomio de segundo grado, ¿cómo se obtiene el término común?

- Con los elementos del trinomio de segundo grado, ¿cómo se obtiene el término lineal?

- Con los elementos del trinomio de segundo grado, ¿cómo se obtiene la constante?

Con el razonamiento anterior, se establece una regla para factorizar los trinomios de segundo grado:

$$
x^{2}+7 x+12
$$

Paso 1. Se abren dos paréntesis:

$$
x^{2}+7 x+12=(\quad)(\quad)
$$

Paso 2. Se extrae raíz cuadrada al término cuadrático y esta se coloca al principio de cada paréntesis.

$$
\sqrt{x^{2}}=x, \quad x^{2}+7 x+12=(x+\quad)(x+\quad)
$$



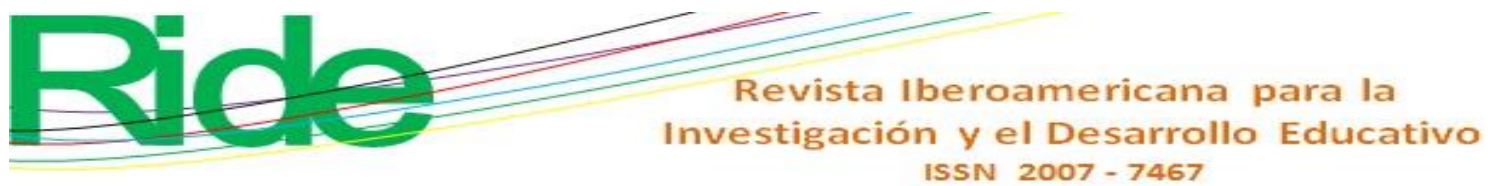

Paso 3. Se buscan dos números que sumados den el término lineal y los mismos multiplicados entre sí sea igual a la constante.

$$
\begin{aligned}
& \left(\begin{array}{l}
3 \\
)
\end{array}\right)+\left(\begin{array}{ll}
4 \\
)
\end{array}\right)=7 \\
& (3)(4)=12
\end{aligned}
$$

Paso 4. Estos números de color rojo se colocan en los paréntesis, y quedan de la siguiente forma:

$$
x^{2}+7 x+12=(x+3)(x+4)
$$

Con el procedimiento anterior, factoriza los trinomios de segundo que se indican a continuación:

$$
\begin{gathered}
4 x^{2}+14 x+12,9 x^{2}+27 x+20,9 x^{2}+9 x+2,49 x^{2}+56 x-20,4 x^{2}-8 x-45 \\
36 x^{2}+6 x-6,25 x^{2}+20 x+3,9 x^{2}+36 x+20,64 x^{2}+24 x-10,100 x^{2}-60 x+8
\end{gathered}
$$

\section{Resultados de la intervención}

Se pueden considerar de buenos a excelentes, puesto que los estudiantes normalistas comprendieron los dos temas, productos notables y factorización, que se estudian en el primer bloque del tercer grado en telesecundaria. Si se toman en cuenta las competencias matemáticas en la educación secundaria:

a) Resolver problemas de manera autónoma.

b) Comunicar información matemática.

c) Validar procedimientos y resultados.

d) Manejar técnicas eficientemente.

Las cuatro competencias matemáticas se lograron con los alumnos normalistas. 
Tabla 1. Rúbrica de productos notables y factorización

\begin{tabular}{|c|c|c|c|c|}
\hline \multirow{2}{*}{$\begin{array}{l}\text { Criterios de } \\
\text { evaluación }\end{array}$} & \multicolumn{4}{|c|}{ Niveles de desempeño } \\
\hline & Excelente & Bueno & Suficiente & Insuficiente \\
\hline $\begin{array}{c}\text { Resolver } \\
\text { problemas de } \\
\text { manera } \\
\text { autónoma }\end{array}$ & $\begin{array}{l}\text { - Realiza alge- } \\
\text { braicamente } \\
\text { operaciones de } \\
\text { multiplicación } \\
\text { y división. } \\
\text { - Maneja las le- } \\
\text { yes de los ex- } \\
\text { ponentes. } \\
\text { - Resuelve pro- } \\
\text { blemas de pro- } \\
\text { ductos notables } \\
\text { y factorización. } \\
\text { Construye las } \\
\text { fórmulas para } \\
\text { su aplicación en } \\
\text { donde corres- } \\
\text { ponda. }\end{array}$ & 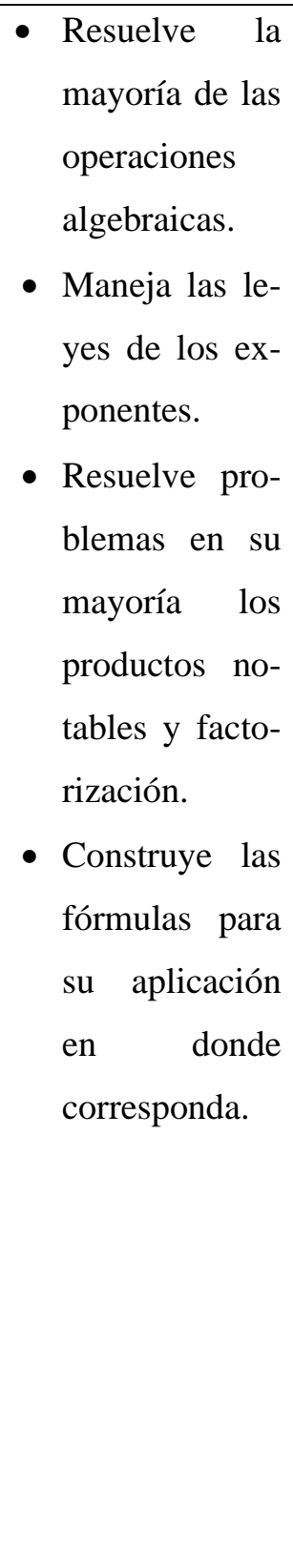 & 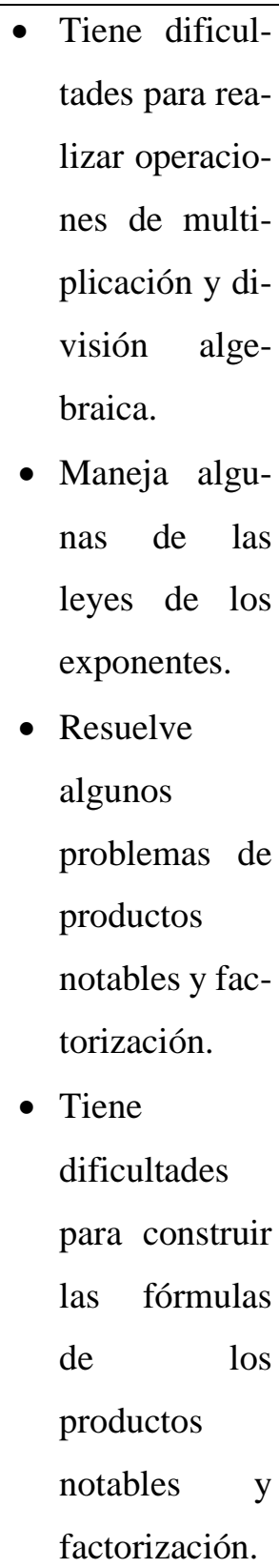 & 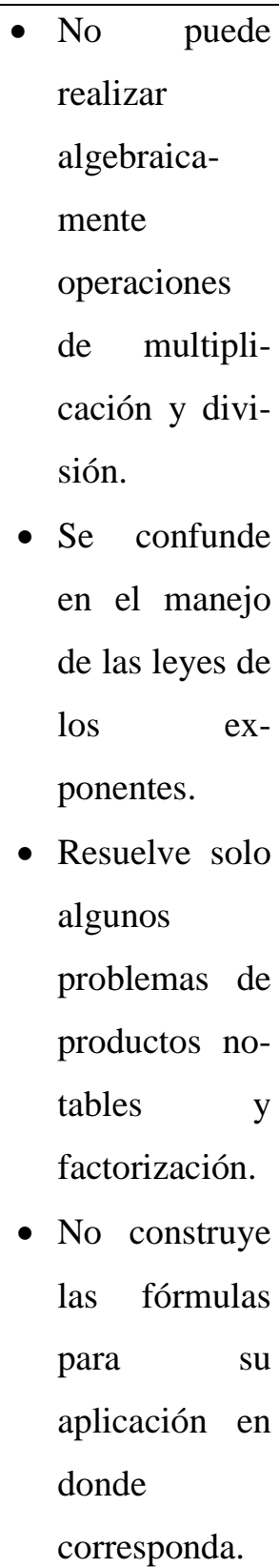 \\
\hline Criterios de & \multicolumn{4}{|c|}{ Niveles de desempeño } \\
\hline evaluación & Excelente & Bueno & Suficiente & Insuficiente \\
\hline $\begin{array}{l}\text { Comunicar } \\
\text { información } \\
\text { matemática }\end{array}$ & 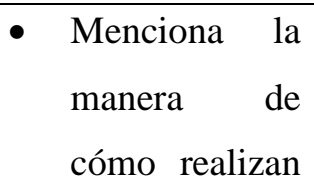 & $\begin{array}{l}\text { - Menciona la } \\
\text { manera de } \\
\text { cómo realiza }\end{array}$ & $\begin{array}{l}\text { - Menciona la } \\
\text { manera de } \\
\text { cómo realiza }\end{array}$ & 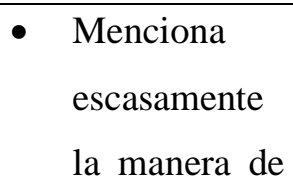 \\
\hline
\end{tabular}




\begin{tabular}{|c|c|c|c|c|}
\hline & $\begin{array}{l}\text { las } \\
\text { operaciones de } \\
\text { las leyes de los } \\
\text { exponentes. } \\
\text { - Comunica la } \\
\text { forma de resol- } \\
\text { ver los bino- } \\
\text { mios cuadra- } \\
\text { dos, binomios } \\
\text { conjugados y } \\
\text { binomios con } \\
\text { un término co- } \\
\text { mún. } \\
\text { Comunica la } \\
\text { manera de } \\
\text { cómo se facto- } \\
\text { rizan los pro- } \\
\text { ductos de los } \\
\text { binomios antes } \\
\text { mencionados. }\end{array}$ & $\begin{array}{l}\text { las operacio- } \\
\text { nes de las le- } \\
\text { yes de los } \\
\text { exponentes. } \\
\text { - Comunica la } \\
\text { forma de re- } \\
\text { solver los bi- } \\
\text { nomios cua- } \\
\text { drados, bino- } \\
\text { mios conju- } \\
\text { gados y bi- } \\
\text { nomios con } \\
\text { un término } \\
\text { común. } \\
\text { Comunica la } \\
\text { manera de } \\
\text { cómo } \\
\text { factorizar } \\
\text { algunos de los } \\
\text { binomios } \\
\text { antes men- } \\
\text { cionados. }\end{array}$ & $\begin{array}{l}\text { las operacio- } \\
\text { nes de algu- } \\
\text { nas leyes de } \\
\text { los exponen- } \\
\text { tes. } \\
\text { - Comunica la } \\
\text { forma de re- } \\
\text { solver de al- } \\
\text { gunos bino- } \\
\text { mios. } \\
\text { Comunica la } \\
\text { manera de } \\
\text { cómo se fac- } \\
\text { torizan los } \\
\text { productos de } \\
\text { algunos bino- } \\
\text { mios antes } \\
\text { mencionados. }\end{array}$ & $\begin{array}{l}\text { cómo } \\
\text { realizan las } \\
\text { operaciones } \\
\text { de las leyes } \\
\text { de los } \\
\text { exponentes. } \\
\text { Comunica en } \\
\text { forma } \\
\text { confusa la } \\
\text { forma de re- } \\
\text { solver los } \\
\text { binomios. } \\
\text { Tiene } \\
\text { dificultades } \\
\text { para comu- } \\
\text { nicar lo que } \\
\text { realiza. }\end{array}$ \\
\hline Criterios de & \multicolumn{4}{|c|}{ Niveles de desempeño } \\
\hline evaluación & Excelente & Bueno & Suficiente & Insuficiente \\
\hline $\begin{array}{c}\text { Validar } \\
\text { información } \\
\text { matemática }\end{array}$ & 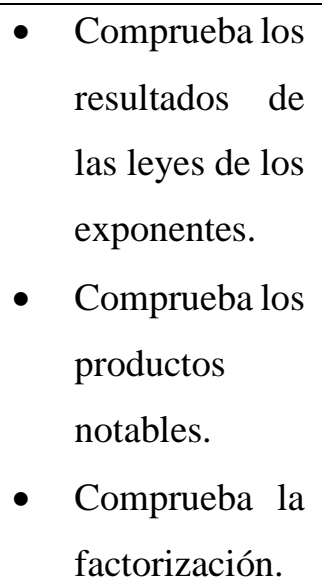 & 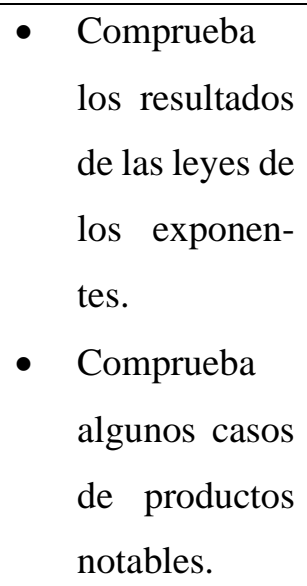 & $\begin{array}{l}\text { - Comprueba } \\
\text { los resultados } \\
\text { de algunos } \\
\text { casos de las } \\
\text { leyes de los } \\
\text { exponentes. }\end{array}$ & \begin{tabular}{lr} 
- & \multicolumn{2}{l}{ Tiene dificul- } \\
tades para \\
comprobar \\
los resultados \\
de las leyes \\
de \\
exponentes. \\
- Tiene dificul- \\
tades para
\end{tabular} \\
\hline
\end{tabular}




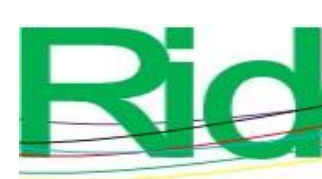

Revista Iberoamericana para la Investigación y el Desarrollo Educativo ISSN 2007 - 7467

\begin{tabular}{|c|c|c|c|c|}
\hline & $\begin{array}{l}\text { Comprueba los } \\
\text { productos notables } \\
\text { y la factorización } \\
\text { por métodos geo- } \\
\text { métricos. }\end{array}$ & 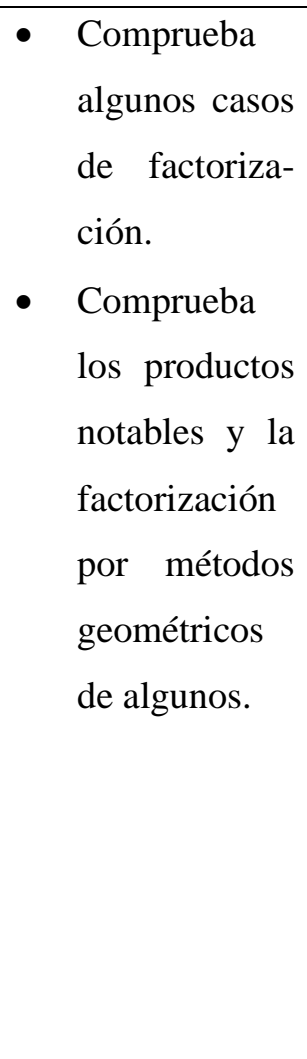 & 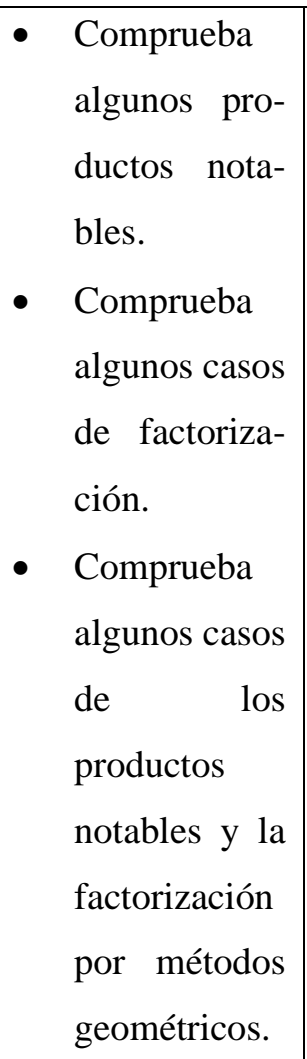 & $\begin{array}{l}\text { comprobar } \\
\text { los productos } \\
\text { notables. } \\
\text { - Escasamente } \\
\text { comprueba la } \\
\text { factorización. } \\
\text { Carece de } \\
\text { compro- } \\
\text { bación de los } \\
\text { productos } \\
\text { notables y la } \\
\text { factorización } \\
\text { por métodos } \\
\text { geométricos. }\end{array}$ \\
\hline Criterios de & \multicolumn{4}{|c|}{ Niveles de desempeño } \\
\hline evaluación & Excelente & Bueno & Suficiente & Insuficiente \\
\hline $\begin{array}{c}\text { Manejar } \\
\text { técnicas } \\
\text { eficientemente }\end{array}$ & 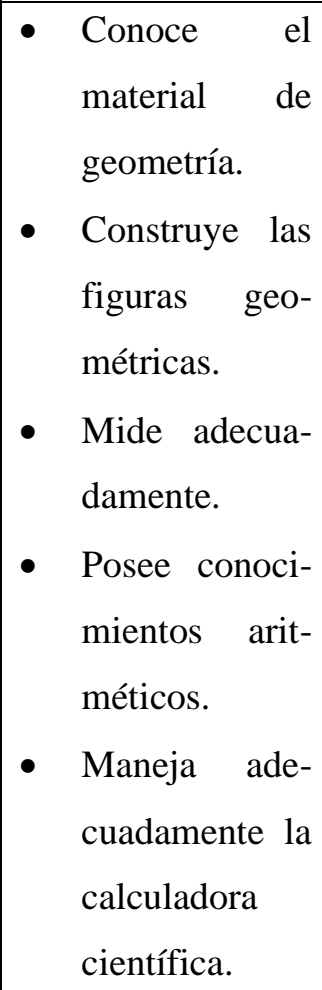 & 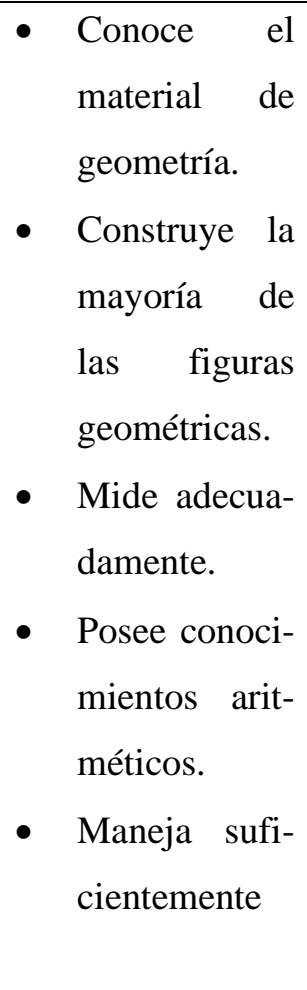 & 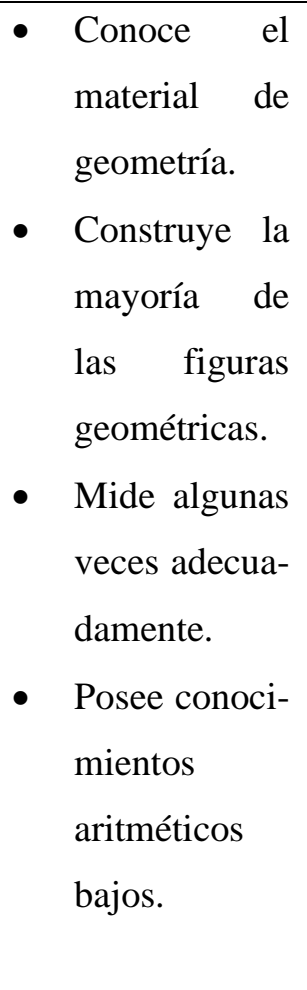 & 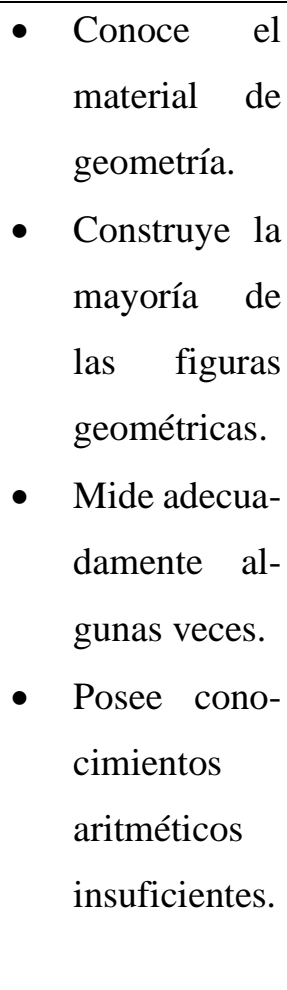 \\
\hline
\end{tabular}




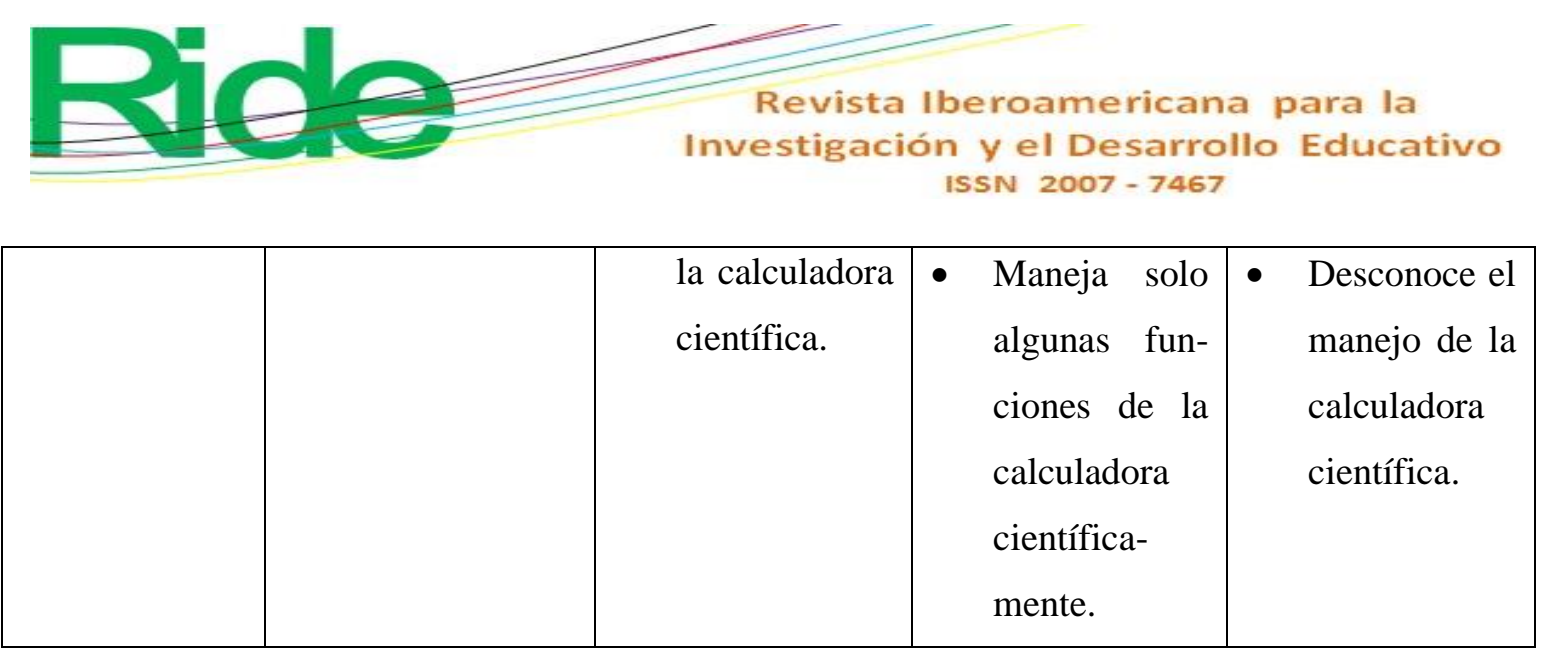

Fuente: Elaboración propia

\section{Discusión}

El objetivo del trabajo de Osorio (2008), a saber, "encontrar significado a las relaciones de equivalencia entre las expresiones que refiere a la medida de las figuras (congruencias entre longitudes, equivalencia entre magnitudes) y comprender esos significados" (p. 3), lo llevó al diseño de materiales manipulables para que sus estudiantes modelaran las figuras geométricas. Mientras que en este trabajo el procedimiento fue vincular la parte geométrica con la algebraica, después obtener el producto de varias parejas de binomios, para llegar a construir la regla que regirá y así obtener el producto.

Gómez (2015), por su parte, afirma que el estudiante aprende a factorizar cuando se implementa el texto paralelo y la resolución de problemas, puesto que "expresa por escrito lo comprendido en su lectura, soluciona y resuelve problemas de factorización” (p. 5).

En cuanto a los productos notables, Barreto (2009) se ocupa de figuras geométricas como cuadrados y rectángulos para representar a los binomios que se multiplicarán para calcular el área.

Hay trabajos cuyo enfoque es análogo al aquí aplicado pero puesto en práctica en otros contextos. Por ejemplo, para Graciano y Aké (2017):

El interés es la búsqueda de aportaciones para la formación de profesores y la mejora de la práctica en el aula. Al respecto, los resultados que se obtuvieron a través de un cuestionario de respuesta abierta rescatan inconsistencias principalmente en el conocimiento especializado del contenido de productos notables, pero también se reconocen áreas de oportunidad para su fortalecimiento (p. 1320). 


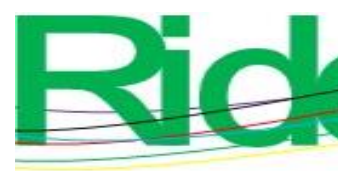

Revista Iberoamericana para la Investigación y el Desarrollo Educativo ISSN 2007 - 7467

producto), se obtiene el binomio cuadrado; lo mismo sucede con los demás binomios: al obtener su producto y después factorizarse, se regresa a la primera expresión algebraica.

No solo comprendieron a los productos notables desde el punto de vista algebraico, sino también geométrico y en su forma verbal; esto significa que se lograron las competencias matemáticas, tal y como se mencionan en el programa de estudios.

Por otra parte, fue interesante que contestaran al principio de cada binomio lo que ellos sabían del tema y después contrastaran esto con lo aprendían al final de la secuencia. Uno de los contenidos que les resultó de mayor facilidad en la obtención de la regla fue el de los binomios conjugados, puesto que solo multiplicaban los términos comunes y simétricos para llegar al resultado, el cual también tiene su nombre específico, diferencia de cuadrados.

Los binomios con los que tuvieron mayor dificultad fueron los que poseen un término común, estos producen un trinomio de segundo grado con características diferentes a los anteriores.

Desde el punto de vista personal, es importante que se continúen haciendo este tipo de trabajos para beneficio de los alumnos. La función que se realizó de parte de quien diseñó esta secuencia fue la de facilitador del aprendizaje: los estudiantes normalistas fueron constructores de su saber, de tal manera que al final de cada caso, concluyeron con la regla que ayuda a resolver los productos o la factorización.

Lo importante de esta secuencia es el razonamiento que se genera al trabajar con los estudiantes de manera grupal, por equipos y de manera individual; se genera un ambiente de aprendizaje favorable para que los estudiantes pregunten con la confianza debida, porque se sabe que ellos serán los futuros profesores.

Por último, las futuras líneas de investigación se pueden dar en el planteamiento de problemas que impliquen el uso de los productos notables y la factorización, con la finalidad de resolver problemas profesionales desde el punto de vista de la economía. 


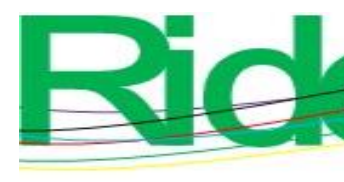

Revista Iberoamericana para la Investigación y el Desarrollo Educativo ISSN 2007 - 7467

\section{Referencias}

Alarcón, J. (coord.) (2005). El libro para el maestro. Matemáticas. Educación secundaria. México: Secretaría de Educación Pública.

Ángeles, J., Guerrero, R. y Loyola, E. (2013). Matemáticas 3. Habilidades y competencias. Ciudad de México, México: Ángeles Editores.

Barreto, J. (2009). Percepción geométrica de los productos notables y de la media geométrica. España: Editorial Números. Revista de Didáctica de Las Matemáticas. pp. 71, 57-74. del Carmen, V., Alfonzo, I. y Trejo, J. H. (2016). El ABC de las tecnologías educativas: tecnologías educativas para las ciencias naturales. México: Centro Regional de Formación Docente e Investigación Educativa.

Gómez, A. (2015). Texto paralelo y aprendizaje significativo de la factorización. (Tesis de licenciatura). Universidad Rafael Landívar, Quetzaltenango.

Graciano, J. y Aké, L. (2017). Conocimiento común y especializado de productos notables de los futuros profesores de matemáticas. En Serna, L. A. (ed.), Acta latinoamericana de matemática educativa (pp. 1320-1329). Ciudad de México, México: Comité Latinoamericano de Matemática Educativa.

Duval, R. (2016). Un análisis cognitivo de problemas de comprensión en el aprendizaje de las matemáticas. En Duval, R. y Sáen, A. (eds.), Comprensión y aprendizaje en matemáticas: perspectivas semióticas seleccionadas (pp. 61-94). Colombia: Universidad Distrital Francisco José de Caldas. Recuperado de http://die.udistrital.edu.co/publicaciones/capitulos_de_libro/un_analisis_cognitivo_d e_problemas_de_comprension_en_el_aprendizaje.

Maldonado, M. (2007). El trabajo colaborativo en el aula universitaria. Laurus, 13(23), 263278. Recuperado de https://www.redalyc.org/pdf/761/76102314.pdf.

Méndez, T. y Cruz, L. (2008). Dificultades en la práctica de productos notables y factorización. Revista del Instituto de Matemática y Física, 11(15), 59-69.

Navarro, J. (2018). Visiones de la realidad educativa. Guadalajara, México: Centro de Investigaciones Pedagógicas y Sociales.

Osorio, G. M. (2008). El álgebra geométrica como recurso didáctico en la enseñanzaaprendizaje del álgebra escolar. Ponencia presentada en el 9. ${ }^{\circ}$ Encuentro Colombiano de Matemática Educativa. Valledupar, del 16 al 18 de octubre de 2008. 

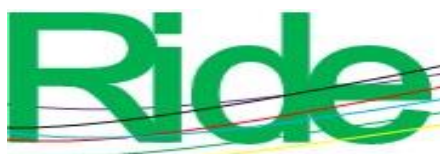

Rico, L. (1998). Errores y dificultades en el aprendizaje de las matemáticas. En Kilpatrick, J., Rico, L. y Gómez, P. (eds.), Educación matemática. Errores y dificultades de los estudiantes. Resolución de problemas. Evaluación. Historia (pp. 69-108). Bogotá, Colombia: una empresa docente- Universidad de los Andes.

Secretaría de Educación Pública [SEP]. (2017). Aprendizajes clave para la educación integral. Plan y programas de estudio para la educación básica. México: Secretaría de Educación Pública.

Socas, M. (2011). La enseñanza del álgebra en la educación obligatoria. Aportaciones de la investigación. Números. Revista de Didáctica de las Matemáticas, 77, 5-34.

Steven, C. (2013). El geómetra. Guía de aprendizaje. Estados Unidos: Key Curriculum Press.

Tobón, S. (2017). El proyecto de enseñanza. Aprendizaje y evaluación. Cuernavaca, México: Centro Universitario CIFE. 